\title{
Unexpectedly high incidence of venous thromboembolism after arthroscopic anterior cruciate ligament reconstruction: prospective, observational study
}

\author{
Masaki Nagashima, ${ }^{1,2}$ Toshiro Otani, ${ }^{3}$ Kenichiro Takeshima, ${ }^{2}$ Hiroyuki Seki, ${ }^{1,2}$ \\ Masanori Nakayama, ${ }^{1,2}$ Nobuto Origuchi, ${ }^{4}$ Ken Ishii ${ }^{1,2}$
}

'Department of Orthopaedic Surgery, International University of Health and Welfare Mita Hospital, Minato-ku, Tokyo, Japan

${ }^{2}$ Department of Orthopaedic Surgery, School of Medicine, International University of Health and Welfare, Narita, Chiba, Japan

${ }^{3}$ Institute for Integrated Sports Medicine, Keio University School of Medicine, Shinjuku-ku, Tokyo, Japan

${ }^{4}$ Department of Vascular Surgery, International University of Health and Welfare Mita Hospital, Minato-ku, Tokyo, Japan

Correspondence to Dr Masaki Nagashima, Department of Orthopaedic Surgery, International University of Health and Welfare Mita Hospital, Minato-ku, Tokyo 1088329, Japan;

masakin@iuhw.ac.jp

Accepted 17 January 2020 Published Online First 26 February 2020

Check for updates

(C) International Society of Arthroscopy, Knee Surgery and Orthopaedic Sports Medicine 2020. No commercial re-use. See rights and permissions. Published by BMJ.

To cite: Nagashima $\mathrm{M}$ Otani T, Takeshima K, et al. J ISAKOS 2020:5:80-82.

\section{ABSTRACT}

Objectives The objectives of this study were to investigate the incidence of deep venous thrombosis (DVT) and pulmonary embolism (PE) after anterior cruciate ligament reconstruction ( $A C L R$ ) using ultrasonography (US) and contrast-enhanced CT (CECT) without pharmacological prophylaxis and to identify the risk factors for DVT.

Methods A prospective, observational study of 55 consecutive Japanese patients undergoing ACLR, including 10 revision surgeries, between April 2017 and September 2018 was performed. All operations were performed by the one experienced surgeon with the use of a tourniquet. Anterior cruciate ligaments were reconstructed using a single-bundle hamstring autograft. US of the leg veins was performed on the sixth or seventh postoperative day. When the patient was diagnosed with DVT, CECT was performed to detect PE. The patients were divided into two groups, those with and those without DVT. Clinical factors were compared between the two groups.

Results After the ACLR, DVT was detected in nine $(16.4 \%)$ patients, and CECT showed that four of them had PE (incidence, at least 7.3\%). All of them were asymptomatic. The mean age was significantly higher in patients with DVT $(41.9 \pm 15.7$ years) than in patients without DVT (28.2 \pm 14.2 years, $p=0.012)$. There were no significant differences in other clinical factors, including sex, body mass index, current smoker, preoperative Lysholm score, time interval from injury to $A C L R$, primary or revision $A C L R$, preoperative knee pain, operative and tourniquet times, and with or without meniscus repair between patients with DVT and those without DVT. Conclusion The incidences of DVT and PE after ACLR were $16.4 \%$ and at least $7.3 \%$, respectively. These incidences, especially PE, were unexpectedly high and might suggest a need for thromboprophylaxis. Since advanced age was identified as the risk factor for DVT in this study, patients with this risk factor should be considered for pharmacological prophylaxis after ACLR. Level of evidence Level IV.

\section{INTRODUCTION}

Arthroscopically assisted anterior cruciate ligament reconstruction (ACLR) is commonly performed for the treatment of anterior cruciate ligament (ACL) injury. Venous thromboembolism, which includes deep venous thrombosis (DVT) and pulmonary embolism (PE), is a complication following
What are the new findings

- The incidences of DVT and PE after ACLR were investigated using ultrasonography and contrast-enhanced CT without pharmacological prophylaxis. The incidences of asymptomatic DVT and PE were revealed to be $16.4 \%$ and at least $7.3 \%$, respectively.

- These incidences, especially PE, were unexpectedly high and might suggest a need for thromboprophylaxis.

- Advanced age was identified as the risk factor for DVT after ACLR. Patients with advanced age should be considered for pharmacological prophylaxis after ACLR.

ACLR. DVT has the potential to develop into life-threatening PE. Since the reported incidence of DVT following ACLR varies from $0.3 \%$ to $41.2 \%{ }^{1-8}$ depending on different modalities used in the study and whether asymptomatic or symptomatic DVT was assessed, the true incidence of DVT after ACLR has not been well clarified. The incidence of symptomatic PE was reported to be $0.05 \%-0.21 \%$ from a large database analysis, ${ }^{1-3}$ but there have only been a few investigations of asymptomatic PE. Therefore, although pharmacological prophylaxis is reported to be effective for reducing DVT after ACLR, ${ }^{9}$ whether pharmacological prophylaxis is routinely needed after ACLR remains controversial. Compared with hip and knee arthroplasties, there is not enough available evidence regarding the prevention of DVT and PE following ACLR.

The objectives of this study were to identify the incidences of DVT and PE after ACLR using ultrasonography (US) and contrast-enhanced CT (CECT) without pharmacological prophylaxis and to identify the risk factors for DVT.

\section{METHODS}

Subjects

This was a prospective, observational study. Between April 2017 and September 2018, 56 consecutive ACLRs of 56 patients, including 10 revision surgeries, were performed at our hospital. All of them were enrolled in this study except for one patient who had a history of PE. 


\section{Surgical procedures and postoperative care}

All ACLRs were performed by one experienced surgeon, with the patient under general anaesthesia with femoral and sciatic nerve blocks and tourniquet use. ACLs were reconstructed using single-bundle hamstring autografts. For graft preparation, a commercially available polyester tape (Leeds-Keio artificial ligament; Neoligament, Leeds, England, UK) was connected at the distal end of the graft and an Endobutton CL BTB (Smith \& Nephew, Andover, Massachusetts, USA) was attached at the proximal end of the graft. The graft was introduced through each tibial tunnel and the femoral tunnel, which was made with transportal technique. The fixation of the graft was performed with the Endobutton on the femoral side and two staples on the tibial side. In revision ACLR, the hamstring autograft was harvested from the contralateral side. If meniscus tears existed and were repairable, they were repaired with FAST-FIX 360 (Smith \& Nephew). A closed-suction drain was installed and left in the knee for 24 hours. Postoperatively, the knee was immobilised with brace for 1 day for patients without meniscus repair and for 5 days for patients with meniscus repair. After the knee immobilisation, range-of-motion exercise and muscle strengthening were started. On the seventh postoperative day, full weight-bearing with hinged knee brace was permitted. Although no patients underwent pharmacological prophylaxis, intermittent pneumatic compression was used for 1 day, and compression stockings were used for 7 days after ACLR.

\section{Diagnosis of DVT and PE}

US of both legs was performed preoperatively and on the sixth or seventh postoperative day to detect DVT. DVT was diagnosed using both compression technique and colour Doppler evaluation. ${ }^{10}$ If the patient was diagnosed with DVT, CECT was performed to detect PE and DVT. US was performed and evaluated by one experienced vascular surgeon. CECT was evaluated by a well-experienced radiologist.

\section{Statistical analysis}

The patients were divided into two groups, those with and those without DVT after ACLR. Preoperative clinical and surgical data, including age, sex, body mass index (BMI), current smoker, Lysholm score, time interval from injury to ACLR, primary or revision ACLR, knee pain, operative and tourniquet times, and with or without meniscus repair, were recorded. Preoperative affected knee pain during walking was assessed using a numerical rating scale (NRS $0-10$ ), and an NRS score of $\geq 3$ was defined as positive for knee pain. These clinical factors were compared between the two groups using the Student t-test, the Mann-Whitney U-test and the $\chi^{2}$ test. All statistical analyses were performed with BellCurve for Excel (Social Survey Research Information Co., Tokyo, Japan).

\section{RESULTS}

\section{Demographic and surgical characteristics of patients}

Of all 55 patients, there were 28 female and 27 male Japanese patients. The mean age, BMI and preoperative Lysholm score were $30.5 \pm 15.0$ years (range 12-63 years), $22.6 \pm 2.5 \mathrm{~kg} / \mathrm{m}^{2}$ and $71.1 \pm 13.3$, respectively. Of the 55 patients, 4 were current smokers and 22 underwent ACLR less than 3 months after the injury. Nine patients had preoperative knee pain during walking. The mean operative and tourniquet times were $80.8 \pm 18.5 \mathrm{~min}$ and $68.5 \pm 14.7 \mathrm{~min}$, respectively. Meniscus repair was performed in 20 patients. The mean operative time of primary ACLR was $74.4 \pm 11.1 \mathrm{~min}$, which was significantly shorter than that of revision ACLR $(109.8 \pm 18.6 \mathrm{~min}, \mathrm{p}<0.001)$.

\begin{tabular}{|c|c|c|c|c|}
\hline \multicolumn{2}{|l|}{ Clinical factor } & DVT (-) & DVT $(+)$ & $P$ value \\
\hline \multicolumn{2}{|l|}{ Age (years) } & $28.2 \pm 14.2$ & $41.9 \pm 15.7$ & 0.012 \\
\hline \multirow[t]{2}{*}{ Sex } & Female & 25 & 3 & 0.249 \\
\hline & Male & 21 & 6 & \\
\hline \multicolumn{2}{|l|}{$\mathrm{BMI}\left(\mathrm{kg} / \mathrm{m}^{2}\right)$} & $22.3 \pm 2.3$ & $23.8 \pm 3.1$ & 0.102 \\
\hline \multirow[t]{2}{*}{ Current smoker } & $(-)$ & 42 & 9 & 0.358 \\
\hline & $(+)$ & 4 & 0 & \\
\hline \multicolumn{2}{|c|}{ Preoperative Lysholm score } & $71.4 \pm 13.9$ & $69.3 \pm 12.4$ & 0.675 \\
\hline \multirow{2}{*}{$\begin{array}{l}\text { Time interval from } \\
\text { injury to } A C L R\end{array}$} & $<3 \mathrm{M}$ & 19 & 3 & 0.655 \\
\hline & $\geq 3 \mathrm{M}$ & 27 & 6 & \\
\hline \multirow[t]{2}{*}{ ACLR } & Primary & 38 & 7 & 0.731 \\
\hline & Revision & 8 & 2 & \\
\hline \multirow[t]{2}{*}{ Knee pain } & $(-)$ & 39 & 7 & 0.603 \\
\hline & $(+)$ & 7 & 2 & \\
\hline \multicolumn{2}{|c|}{ Operative time (min) } & $80.4 \pm 19.0$ & $83.1 \pm 17.7$ & 0.691 \\
\hline \multicolumn{2}{|c|}{ Tourniquet time (min) } & $67.8 \pm 14.6$ & $71.9 \pm 16.2$ & 0.455 \\
\hline \multirow[t]{2}{*}{ Meniscus repair } & $(-)$ & 27 & 8 & 0.085 \\
\hline & $(+)$ & 19 & 1 & \\
\hline
\end{tabular}

$\mathrm{ACLR}$, anterior cruciate ligament reconstruction; BMI, body mass index; DVT, deep venous thrombosis.

\section{Incidences of DVT and PE}

Although no DVT was detected before ACLR, DVT was detected in nine patients (16.4\%) postoperatively, of whom four had PE (incidence, at least 7.3\%). All patients with DVT and/or PE were asymptomatic. In eight of nine patients, DVT was found in the injured side and was located at the soleal vein in five patients, at the peroneal vein in one patient, at the soleal and peroneal veins in one patient, and in the soleal, peroneal, posterior tibial and popliteus veins in one patient. In one patient undergoing revision ACLR, the DVT was found in both the soleal and peroneal veins of both legs. The patients with DVT and/or PE were treated with anticoagulant therapy with edoxaban or apixaban. When comparing patients with DVT to patients without DVT, age was significantly higher in patients with DVT $(\mathrm{p}=0.012)$ (table 1).

\section{DISCUSSION}

In the present study, the incidences of asymptomatic DVT and PE were $16.4 \%$ and at least $7.3 \%$, respectively. Asymptomatic PE was found in about half of the patients with DVT. Although advanced age was shown to be the risk factor for DVT after ACLR, other factors, such as preoperative knee pain, revision ACLR and meniscus repair, were not significantly associated with DVT development. Based on a large database analysis, the incidence of DVT after ACLR was reported to be very low, ranging from $0.30 \%$ to $0.34 \% \cdot{ }^{1-3}$ In contrast, in a study that used venography to examine for DVT, the incidence was reported to be $14.0 \%,{ }^{4}$ and with US, the incidence was reported to be $9 \%$ and $12.1 \%,{ }^{56}$ almost the same as the present results. Adala et al reported a low incidence of DVT after ACLR (1.8\%) using US, ${ }^{7}$ presumably because they excluded patients above the age of 45 years and with BMI greater than $30 \mathrm{~kg} / \mathrm{m}^{2}$. The incidence of symptomatic PE was reported to be very low, ranging from $0.05 \%$ to $0.21 \%$ from a large database analysis, ${ }^{1-3}$ but there have only been a few investigations of asymptomatic PE. In the present study, the incidence of asymptomatic PE was unexpectedly high. There are reports of cases with fatal PE after ACLR. ${ }^{11}{ }^{12}$ Thus, the prevention of DVT and PE after ACLR should be considered an urgent issue.

Pharmacological prophylaxis, such as low-molecular-weight heparin (LMWH), is reported to be effective for reducing DVT 
after knee arthroscopy ${ }^{813}$ and ACLR. ${ }^{9}$ However, whether the benefits of LMWH outweigh the risks and costs remains controversial. ${ }^{14}$ According to the American College of Chest Physicians guidelines, pharmacological prophylaxis was not suggested for patients without histories of DVT and PE. ${ }^{15}$ In fact, there is no consensus regarding the indication for pharmacological prophylaxis after ACLR.

In the present study, age was identified as a risk factor for DVT after ACLR. Similarly, Ye et al used venography to examine for DVT after ACLR and reported that age older than 35 years was a risk factor, ${ }^{4}$ and Struijk-Mulder et al used US to screen for DVT after ACLR and reported that advanced age was a risk factor. ${ }^{5}$ Jameson et al and Gaskill et al identified the incidence of DVT after ACLR based on a large database and reported the risk factor to be age older than $40^{1}$ and 35 years, ${ }^{2}$ respectively. In addition to age, prolonged tourniquet time and multiple ligament reconstructions have been reported as risk factors for DVT after ACLR. ${ }^{6}$ Patients with advanced age and other risk factors should be considered for pharmacological prophylaxis following ACLR. Further studies are needed to determine the optimal indication for pharmacological prophylaxis and to develop other thromboprophylactic approaches.

The ideal duration of knee immobilisation after ACL reconstruction has not been elucidated. In previous studies, the reported duration was varied from 0 to 7 days. ${ }^{4716}$ In our study, the operated knee was immobilised for 1 or 5 days, which is not much different from these reports. Camp et al investigated the effect of the timing of the initiation of mechanical loading after ACL reconstruction in a murine model and reported that knees that were immobilised for 5 days demonstrated greater biomechanical strength and superior histological evidence of healing compared with knees that were allowed immediate motion or that were immobilised for more than 10 days. ${ }^{17}$ However, it remains unclear how these findings can be translated to clinical practice. The longer the duration of knee immobilisation, the higher the risk of DVT and PE. Further research is needed to clarify the ideal duration in a clinical population.

It is controversial whether to use the intra-articular drain after ACLR. In our hospital, the drain was routinely used to minimise postoperative knee pain and swelling after ACLR, and there were some reports with the use of the intra-articular drain after ACLR. ${ }^{516}$ However, there are some problems that include a possibility of infection and cost by using the drain. Additional studies are necessary to evaluate the necessity of the drain after ACLR.

This study has some limitations. First, it included a small number of patients. Although the sample size of our observational study was planned to set at about 100 cases initially, similar to that conducted by Struijk-Mulder et al, ${ }^{5}$ since the incidence of PE was unexpectedly high, we considered that we had to stop this study and report the results. Currently, in patients more than 40 years of age, pharmacological prophylaxis after ACLR and no tourniquet use during ACLR are considered in our hospital. Second, since CECT was performed only for patients found to have DVT after ACLR, not all patients underwent CECT. Since cases of symptomatic PE without DVT following ACLR have been reported, ${ }^{11} 12$ the incidence of PE in this study might still have been underestimated.

\section{CONCLUSION}

The incidences of DVT and PE after ACLR were investigated using US and CECT without pharmacological prophylaxis. The present study showed that the incidences of asymptomatic DVT and PE were $16.4 \%$ and at least $7.3 \%$, respectively. These incidences, especially PE, were unexpectedly high and might suggest a need for thromboprophylaxis. Since advanced age was identified as the risk factor for DVT in this study, patients with this risk factor should be considered for pharmacological prophylaxis after ACLR.

Correction notice The address of the corresponding author has been updated since this article was first published online.

Contributors MNag, TO and NO designed the study. MNag, KT, HS and MNak coordinated and conducted the data collection. MNag, TO, HS and NO provided the data, analysis tools and data analysis. MNag, TO, KT, NO and KI drafted the manuscript. All authors reviewed and approved the manuscript before submission.

Funding The authors have not declared a specific grant for this research from any funding agency in the public, commercial or not-for-profit sectors.

Competing interests None declared.

Patient consent for publication Not required.

Ethics approval This study was approved by the institutional review board, and written informed consent was obtained from all patients.

Provenance and peer review Not commissioned; externally peer reviewed.

Data availability statement All data relevant to the study are included in the article or uploaded as supplementary information. E-mail address of the corresponding author: masakin@iuhw.ac.jp.

\section{REFERENCES}

1 Jameson SS, Dowen D, James P, et al. Complications following anterior cruciate ligament reconstruction in the English NHS. Knee 2012;19:14-19.

2 Gaskill T, Pullen M, Bryant B, et al. The prevalence of symptomatic deep venous thrombosis and pulmonary embolism after anterior cruciate ligament reconstruction. Am J Sports Med 2015:43:2714-9.

3 Kraus Schmitz J, Lindgren V, Janarv P-M, et al. Deep venous thrombosis and pulmonary embolism after anterior cruciate ligament reconstruction: incidence, outcome, and risk factors. Bone Joint J 2019;101-B:34-40.

4 Ye $S$, Dongyang $C$, Zhihong $X$, et al. The incidence of deep venous thrombosis after arthroscopically assisted anterior cruciate ligament reconstruction. Arthroscopy 2013:29:742-7.

5 Struijk-Mulder MC, Ettema HB, Verheyen CCPM, et al. Deep vein thrombosis after arthroscopic anterior cruciate ligament reconstruction: a prospective cohort study of 100 patients. Arthroscopy 2013;29:1211-6.

6 Dong J-tao, Wang X, Men X-qian, et al. Incidence of deep venous thrombosis in Chinese patients undergoing arthroscopic knee surgery for cruciate ligament reconstruction. Knee Surg Sports Traumatol Arthrosc 2015;23:3540-4.

7 Adala R, Anand A, Kodikal G. Deep vein thrombosis and thromboprophylaxis in arthroscopic anterior cruciate ligament reconstruction. Indian J Orthop 2011;45:450-3

8 Marlovits S, Striessnig G, Schuster R, et al. Extended-duration thromboprophylaxis with enoxaparin after arthroscopic surgery of the anterior cruciate ligament: a prospective, randomized, placebo-controlled study. Arthroscopy 2007;23:696-702.

9 Zhu J, Jiang H, Marshall B, et al. Low-Molecular-Weight heparin for the prevention of venous thromboembolism in patients undergoing knee arthroscopic surgery and anterior cruciate ligament reconstruction: a meta-analysis of randomized controlled trials. Am J Sports Med 2019;47:1994-2002.

10 Guideline developed in collaboration with the American College of Radiology, Society of Pediatric Radiology, Society of Radiologists in Ultrasound. AlUM practice guideline for the performance of peripheral venous ultrasound examinations. J Ultrasound Med 2015;34:1-9.

11 Bourget-Murray J, Clarke MA, Gorzitza S, et al. Symptomatic bilateral pulmonary embolism without deep venous thrombosis in an adolescent following arthroscopic anterior cruciate ligament reconstruction: a case report and review of the literature. $J$ Med Case Rep 2018;12:194.

12 Koaban S, Alatassi R, Ahmed B, et al. Bilateral pulmonary embolism after arthroscopic anterior cruciate ligament reconstruction: a case report. Int I Surg Case Rep 2018;49:64-6.

13 Sun Y, Chen D, Xu Z, et al. Deep venous thrombosis after knee arthroscopy: a systematic review and meta-analysis. Arthroscopy 2014;30:406-12.

14 Bushnell BD, Anz AW, Bert JM. Venous thromboembolism in lower extremity arthroscopy. Arthroscopy 2008;24:604-11.

15 Falck-Ytter Y, Francis CW, Johanson NA, et al. Prevention of VTe in orthopedic surgery patients: antithrombotic therapy and prevention of thrombosis, 9th ed: American College of chest physicians evidence-based clinical practice guidelines. Chest 2012;141:e278S-325.

16 Nakayama H, Yoshiya S. The effect of tourniquet use on operative performance and early postoperative results of anatomic double-bundle anterior cruciate ligament reconstruction. J Orthop Sci 2013;18:586-91.

17 Camp CL, Lebaschi A, Cong G-T, et al. Timing of postoperative mechanical loading affects healing following anterior cruciate ligament reconstruction. J Bone Joint Surg Am 2017;99:1382-91. 